\title{
CIUDAD Y MUNDO DE LA VIDA
}

\author{
ENRIQUe España NaVeira \\ Arquitecto. Investigador en OUT_Arquias, Universidad de Sevilla \\ https://dx.doi.org/10.12795/astragalo.2017.i23.11
}

\section{Teoría del mundo de la vida, Hans Blumenberg, Fondo de Cultura Económica (2013). Trad. de Griselda Mársico y Uwe Schoor}

Hoy nos preguntamos algo que demasiadas veces ha sido esquivado al pensar la ciudad, la escurridiza y sigilosa idea de entender la vida como preocupación central para concebir el espacio. La preservación y el cuidado de la socialidad como proyecto constituyente de la ciudad, compuesta por las múltiples formas de vida que se despliegan por cada rincón del territorio. Estas dudas no nacen de la coyuntura de crisis de la última década, pero sí que se están extendiendo gracias a ella. Con la crisis surgen las deslealtades, aparecen las dudas con las creencias hasta ahora inquebrantables, se tambalea el sentido de lo que entendemos como vida y la objetividad de lo que nos rodea. Tiembla la arquitectura del mundo, y bajo el tambaleo aparecen apoyos hasta ahora invisibles.

La ciudad y el mundo de la vida han tenido históricamente apasionantes desencuen- tros, y el libro de Hans Blumenberg, es en este caso una buena excusa, un eslabón perdido, un aprendizaje de partida para trazar algunos reencuentros. Teoría del mundo de la vida es el resultado de la recopilación de cinco manuscritos editados póstumamente pertenecientes a lo que el editor Manfred Sommer (Blumenberg, 2013) nos advierte que el autor clasificó como pertenecientes a la temática de una "teoría del mundo de la vida". Aunque nunca fue pensado realmente como un libro, al menos no como algo acabado. Es importante advertir esta condición fragmentaria e inacabada para poder entender y acercarse mejor a este compendio de textos sobre una teoría del mundo de la vida, en los que destaca un profundo esfuerzo por describir ciertas contradicciones desde un origen fenomenológico. De hecho, como describe el editor, "teoría del mundo de la vida" es una contradicción en sí misma, «En el mundo de 
la vida no puede haber teoría del mundo de la vida. El mundo de la vida es la suma de las condiciones bajo las cuales es absolutamente imposible la teoría» (Blumenberg, 2013: 266). Sin embargo, la contradicción no es un freno para Blumenberg sino todo lo contrario, en esta serie de trabajos el autor entiende que es necesario abordar qué se entiende por "mundo de la vida", una noción todavía muy lejos de tener un significado unívoco y que nos permite adentrarnos en la ambigüedad que supone. Resaltaremos unas partes más que otras para conectarnos de manera profana con otros autores y en otros tiempos para imaginar de qué manera el mundo de la vida se relaciona con la ciudad.

\section{EL HORIZONTE FENOMENOLÓGICO}

Blumenberg se ocupa de recuperar el concepto de "mundo de la vida" [Lebenswelt] que introdujo ${ }^{1}$ Edmund Husserl en 1924 en la fenomenología a partir de un texto sobre Kant ${ }^{2}$ y que siguió desarrollando en trabajos posteriores donde se convierte en un tema central de los escritos husserlianos hasta la elaboración definitiva de Crisis en 1936 (Husserl, 1991). En Husserl abordar el mundo de la vida fue un intento de ofrecer un mejor acceso a la fenomenología. «El mundo de la vida es para Husserl nuestro mundo natural, el mundo en el que vivimos y en el

1 El concepto ya había sido usado por otros filósofos como Georg Simmel en 1906 (el libro es revisado y publicado de nuevo en 1912), en su libro Die Religion diciendo "construir un mundo de la vida autónomo" (statt eine autonome Lebenswelt aufzubauen) (Simmel, 1912: 4).

2 Texto publicado bajo el nombre Kant und die Idee der Traszendentalphilosophie en el libro Erste Philosophie (1923/24). Erster Teil, Gesammelte Werke, Nijhoff, La Haya, 1950 ss, vol. VII, p. 232. que estamos imbuidos en nuestras actividades cotidianas» y desde ese mundo hacernos reflexionar sobre este mundo, «hacernos ver que nosotros lo constituimos» (Follesdal, 1990: 66). Husserl se refería con esto al mundo vivido que todavía no es tematizado por teoría alguna en cuanto es "lo que se da por sentado o supuesto" y que la crítica de la razón de Kant presupone como existente sin más (Durán, 2014: 311). Dentro de un momento de "crisis de las ciencias europeas" (Husserl, 1991), Husserl se preocupa por la experiencia del mundo premodal, de la vida que precede (y al mismo tiempo acompaña) de una forma lógica a la ciencia, es decir, el estado anterior de toda teoría (lo sobreentendido ${ }^{3}$ ). Así Husserl piensa que es posible una ciencia del mundo de la vida. Esto es precisamente lo que niega Blumenberg en Teoría del mundo de la vida, que pueda existir una ciencia del mundo de la vida, de ahí lo paradójico del título.

Blumenberg empezará desde este punto a cuestionar y tensar la complejidad y profundidad de la región conceptual del mundo de la vida. «El mundo de la vida es inestable porque vive de su apariencia de estabilidad y de los paliativos y las astucias que eso requiere» (Blumenberg, 2013: 114). Esta inestabilidad habla también de su ambivalencia, lo sobreentendido funciona como estabilizador en la inercia del mundo de la vida y, al mismo tiempo, supone el ocultamiento de una posible refundamentación del estado de las cosas. No se queda tampoco ahí, en la relación entre cientificidad y precien-

3 «Lo que he llamado la tendencia del mundo de la vida a la inercia de existencia y que en otro contexto llamo su premodalidad es lo que Husserl reiteradamente denomina lo sobreentendido [die Selbstverständlichkeit].» (Blumenberg, 2013: 115-116) 
tificidad despeja de algún modo la idea de que ese estado de premodalidad contiene un fuerte potencial, no capturable por la ciencia, que a su vez necesita la ciencia para desarrollarse, en sus propias palabras: «el mundo de la vida detiene la vida, pero sólo deteniéndola prepara sus movimientos» (Blumenberg, 2013: 115).

El mundo de la vida es el "suelo del mundo dado ya sobreentendidamente por la experiencia", es el "ser del mundo en sus sobreentendidos indudables", que se han vuelto indudables no sin cooperación del tiempo, porque este sobreentendido del mundo de la vida está compuesto en cierto modo por los "sobreentendidos milenarios", que en Descartes y en su crítica habían aparecido como prejuicios, como meros restos de la escolaridad de la filosofía. (Blumenberg, 2013: 118)

Para Husserl, que estaba muy influenciado por el cientificismo de fines de siglo XIX, resultaba muy difícil dar el salto hacia un distanciamiento de la forma científica del pensamiento, la ciencia era el progreso humano por antonomasia (Blumenberg, 2013: 123). Desde la fuerte influencia del neokantismo, pero ya desbordando los límites de la búsqueda del apriori, Ernst Cassirer (1979) busca este apriori «ya por debajo de toda formación de objeto teórica», en relación directa e inmediata con el mundo, donde «el mundo de la vida aparece como la base de todas las condiciones a priori, hasta la ciencia como su último peldaño» (Blumenberg, 2013: 125). Husserl lleva la ampliación de la temática del apriori (neokantiana) a los «juicios sintéticos a priori» ${ }^{4}$, donde el juicio

4 La forma lógica que Kant descubrió y convirtió en la base de su crítica de la razón. (Blumenberg, 2013: 125) analítico se basa en el análisis de la intuición en vez de en el análisis del concepto. Para profundizar en la fundamentación fenomenológica de algunos de los conceptos que estaba desarrollando como el de «vida» o el de «experiencia original», según Blumenberg, Husserl piensa que es necesario problematizar esta cuestión de una forma autónoma (del apriori neokantiano) aplicando el conocido método de la reducción fenomenológica para intentar dilucidar la vieja diferenciación entre essentia y existentia, "para llegar a una ampliación de las determinaciones esenciales» (Blumenberg, 2013: 126). Para ello, el método consistía en dos pasos:

- 1. La reducción de lo que Husserl llama la tesis general, la abstracción de la existencia concreta dentro de un mundo de otros objetos existentes, la limitación del objeto a su mera representabilidad, la indistinción de percepción e imaginación.

- 2. La variación libre, el disponer libremente del objeto imaginado, probar la modificación de sus atributos con vistas a establecer qué resulta ser prescindible y qué necesario.

(Blumenberg, 2013: 126-127)

Esta operación no resultó ser tan simple como parecía y comenzaron las dificultades al preguntarse ¿qué pasa con el espacio?, y avanza Blumenberg: «¿en la reducción fenomenológica el espacio también debe ser sólo un espacio representado o el espacio real, homogéneo, existente queda excluido de la reducción fenomenológica?» Resulta que entrañaba un problema metodológico de no poder reducir a un valor 
unitario de la existencia, de la tesis general, sino que se trataba de algo "temáticamente de una productividad, multiplicidad, heterogeneidad extraordinarias. El objeto reductible a su esencia no existía; todo objeto estaba inserto en un horizonte de relaciones, remisiones, referencias, expectativas, anticipaciones típicas que no era posible eliminar sin intervenir en la propia estructura interna del objeto empobreciéndola» (Blumenberg, 2013: 127). Husserl describió esta situación con el concepto de horizonte, explicando que todo objeto está en un horizonte.

Los horizontes de distintos objetos se superponen, se imbrican, conforman complejos de horizontes de orden superior. El valor límite de los complejos de horizontes y de los órdenes de horizontes está en el concepto de mundo. El mundo es la totalidad de los complejos de horizontes. El mundo tiene su propio tipo y estructura. Es la totalidad que regula todo proceso consistente en atravesar las remisiones de horizontes. Todo objeto está indispensablemente unido a su pertenencia a un mundo, a este mundo. El concepto de mundo es un concepto límite. El mundo es el horizonte de los horizontes. (Blumenberg, 2013: 127-128)

\section{EL REPLIEGUE DE LA CIUDAD}

¿De qué forma se relaciona el mundo de la vida con la ciudad?, ¿qué tiene que ver con este horizonte? Vamos a movernos para descubrir posibles relaciones. Resulta muy interesante analizar un texto y un curso de Gilles Deleuze en los que se abordan y bordean las preguntas de Blumenberg y Husserl de un modo enormemente imaginativo y en ciertos momentos conectado con algunas de sus intuiciones. Empieza diciendo Deleuze, en Los repliegues de la materia, que «el Barroco no remite a una esencia, sino más bien a una función operatoria, a un rasgo. No cesa de hacer pliegues» (Deleuze, 1989: 11). Una vez que aparcamos la búsqueda de la reducción a la essentia, nos podemos centrar en una actitud más abierta, más compleja, que entiende que el valor límite de los complejos de horizontes está en el infinito; en el despliegue infinito de pliegues y repliegues. Leibniz sí supera definitivamente el cartesianismo, en palabras de Deleuze, «si el mundo es infinitamente cavernoso, si hay mundos en los mínimos cuerpos, es porque hay "en todas partes un resorte de la materia", que no sólo habla de la división infinita de las partes, sino de la progresividad de la adquisición y la pérdida de movimientos, sin dejar de realizar la conservación de la fuerza» (Deleuze, 1989: 15). La multiplicidad que aparecía en Husserl nos lleva a desarrollar esta idea sobre los mundos de la vida, no ya solo con la ambigüedad de la que hablaba Blumenberg, sino como multiplicidad; «lo múltiple no sólo es lo que tiene muchas partes, sino lo que está plegado de muchas maneras» (Deleuze, 1989: 11).

En el extenso análisis que hace Deleuze sobre Leibniz, destaca el realizado sobre el punto de vista en relación a la ciudad en su curso llamado El punto de vista. El pliegue, Leibniz y el Barroco, realizado en la Universidad París 8 en noviembre de $1986^{5}$. Deleuze lleva la idea de Husserl de horizonte, "el mundo como horizonte

5 Esta clase titulada El punto de vista. El pliegue, Leibniz y el Barroco, tuvo lugar en la Universidad París 8 en noviembre de 1986 y se puede ver en el siguiente enlace (consultado el 21 de noviembre de 2017): https://www.youtube.com/ watch? $\mathrm{v}=\mathrm{pva0}$ ciKe0fo\&t=6916s (todo lo referente a la ciudad está a partir del momento 1:48:30 del vídeo) 
de los horizontes”, mucho más allá. Enunciará que "cada uno de nosotros somos un punto de vista sobre la serie infinita del mundo", a lo que llama también ciudad. Es decir, que cada uno de nosotros es un punto de vista sobre la ciudad. Un enunciado aparentemente simple que encierra una enorme complejidad. Deleuze hace tres aclaraciones: primero, que es falso que un perfil/cara de la ciudad correspondan cada punto de vista; segundo, que cada punto de vista corresponde la totalidad de la serie; y tercero, que hay una necesidad por la multiplicidad de puntos de vista porque la totalidad de la serie está necesariamente afectada por una suma infinita de variaciones.

Cada sujeto, cada uno de nosotros, comprende el mundo entero como una serie infinita. Lo que no significa que lo comprenda con claridad, esto sucedería a través de una profundidad (en el pliegue). Es en la profundidad que se comprende el mundo entero, la totalidad de la serie. Somos puntos de vista, pero tenemos nuestro propio campo de claridad y oscuridad, vemos unas cosas con claridad y otras oscuras. Aquí Deleuze pone el ejemplo siguiente: “Tienen alma las bestias? Sin duda, las bestias son puntos de vista. El punto de vista de la mariposa, del elefante...". Con esto hace hincapié en las diferentes profundidades, un animal también tiene punto de vista, aunque vea con poca claridad. Es decir, la variedad y multiplicidad de los puntos de vista sería tanta como criaturas existen $y$, al mismo tiempo, estos puntos de vista tendrían profundidad, claroscuros, diferentes formas de claridad y oscuridad. La composición de la ciudad, del mundo, estaría formada por el pliegue, despligue y repliegue de los puntos de vista sobre la ciudad. La ciudad en sí no existe, o al menos no existe fuera de los puntos de vista sobre la ciudad. La ciudad no es un objeto. ¿Qué entendemos entonces por ciudad? La respuesta, según Deleuze, solo puede ser el acuerdo entre puntos de vista sobre la ciudad. Es decir, que la ciudad está siempre plegada, y estar plegada significa estar en/dentro, incluida en el punto de vista. Significa que la ciudad es el acuerdo entre sujetos, entre subjetividades.

¿Qué operaciones se descubren desde este punto de vista? Surge un cuestionamiento sobre el par precientificidad-cientificidad, se pliegan, son puntos de vista con diferente profundidad. Los mundos de la vida emergen y se sumergen, en la multiplicidad de pliegues que la constituyen, a distintos ritmos y con diferentes temporalidades, teoría y praxis, racionalidad y mitos, ritos, gritos, signos, señales, en un sinfín de intensidades. Podemos entender esta composición transversal de acuerdos entre subjetividades como la socialidad imprescindible para hacer ciudad. ¿Hasta qué punto es esto posible hoy? Es decir, ¿qué cambios supondría estar forma de ver y hacer ciudad?

\section{EL ESPACIO VIVIDO}

¿Cómo se podría profundizar en esta forma de hacer ciudad? Una que parta del cuidado de la socialidad, de los cuerpos, de la vida. A partir de aquí vamos a profundizar sobre un autor, Henri Lefebvre, que centró sus reflexiones en otra forma de ver y hacer ciudad partiendo de un profundo análisis sobre el mundo de la vida. En 1946, Henri Lefebvre, comienza un trabajo de largo recorrido que hará en tres volúmenes sobre una Crítica a la vida cotidiana (Lefebvre, 
2014). En el primer volumen se propondrá «llevar la banalidad de lo cotidiano al lenguaje y lo conceptual» (Lefebvre, 1976: 207). Al margen de las diferencias entre los conceptos de mundo de la vida y mundo cotidiano, ya advertidos por Blumenberg (2013: 61), en Lefebvre la cuestión adquiere un carácter revolucionario, es decir, su análisis está centrado en poner en valor la riqueza de lo cotidiano y en la transformación de la vida. Esto quiere decir que no resuelve el atolladero teórico y metodológico que desvela Blumenberg sobre la imposibilidad de que exista una teoría del mundo de la vida, en tanto que mundo calificado por la premodalidad de lo sobreentendido. Es decir, sobre el problema central de la objetividad frente a lo sobreentendido. En Lefebvre se produce un giro marxista que pone en el centro la preocupación por la transformación de la vida, comprender siempre ligado a transformar. Para Lefebvre «sólo llegaríamos a transformar la vida cotidiana mediante una previa penetración en ella a través del análisis, en lugar de evadirnos de ella mediante la poesía (surrealistas) o por la filosofía (filósofos). Así abordé de frente el problema de la vida cotidiana.» (Lefebvre, 1976: 209). Se podría decir que hay un segundo giro, heideggeriano, en el que lo sobreentendido es reformulado como cotidianidad y que es crítico con el método fenomenológico, descrito por Heidegger como «simple ver y retener lo visto, sin la pregunta curiosa de qué hacer con eso» ${ }^{6}$.

En el segundo volumen (1961) de su crítica a la vida cotidiana, después de 15 años de reconstrucción del capitalismo, lo cotidia-

6 Descrito así por Martin Heidegger en el curso de Maburgo del semestre de verano de 1925, citado a su vez por Blumenberg (2013: 116). no aparece empobrecido por la alienación (concepto introducido a raíz del estudio que hace Lefebvre de los primeros textos de Marx, muy cercano al concepto de reificación en Lukács). Lo cotidiano ahora se muestra como el resultado del mundo de la mercancía, arrastrado por la uniformidad del programa del capitalismo y el Estado organizando la vida cotidiana. Aquí es donde se forja la "sociedad de consumo", que Lefebvre anuncia como "sociedad burocrática de consumo dirigido". Esta ambivalencia y multiplicidad de lo cotidiano adquiere en este punto mayor complejidad.

En el tercer volumen (1981) Lefebvre realiza un acercamiento metafilosófico en el que va a hablar sobre lo repetitivo y la diferencia, con la idea de reivindicar el derecho a la diferencia (Lefebvre, 1971) contra el modelo lógico y estructuralista según el cuál la razón triunfaría en lo idéntico, que se sustenta en el reino de la mercancía y el dinero y donde el Estado lo que hace es reducir las diferencias para facilitar este trabajo (por ejemplo la organización del espacio). Frente a los procesos de homogenización Lefebvre propone la diferencia y esta cuestión la enmarca en un debate metafilosófico que él mismo pone en conflicto con Deleuze ${ }^{7}$. Por razones muy distintas su entendimiento de la diferencia es su mayor crítica a Marx, en contra de una realidad homogénea que piensa en la sociedad como totalidad. Para Lefebvre, cotidianidad y diferencia se insertan en el marxismo, pero más allá de Marx. También aportará reflexiones

7 «Deleuze, tiende a separar el reino de lo repetitivo, lo idéntico, y el de la diferencia, el flujo, la corriente, yo intuyo un movimiento dialéctico entre ambos términos, un movimiento conflictivo que tendría lugar en el cuerpo y sus ritmos.» (Lefebvre, 1976: 212). 
sobre el capitalismo y su mundialización (globalización), dentro de esta idea se enmarcará su hipótesis sobre la urbanización planetaria (Lefebvre, 1972) fenómeno que tiende a reducir las diferencias, a homogeneizar a todas las sociedades y reducirlas a un modelo único.

En 1974, Lefebvre escribe La producción del espacio (Lefebvre, 2013), como culminación de un recorrido conceptual para pensar la espacialidad $^{8}$. Lefebvre centra su reflexión en la problemática del espacio a partir de los años 60, convirtiéndose en el autor más importante en enunciar un análisis de la complejidad de la sociedad moderna capitalista desde un enfoque eminentemente urbano. En La producción del espacio, el filósofo francés propone como operación la trialéctica del espacio en la que se divide entre: el espacio de lo percibido (físico), de lo concebido (mental) y de lo vivido (social), fundamental para redefinir conceptualmente la formar de hacer ciudad. En estas división, el espacio percibido sería el de las prácticas espaciales (el espacio de la experiencia material); el espacio concebido sería el de las representaciones del espacio (el espacio de los expertos, los científicos, los planificadores, también de los signos, códigos y la fragmentación); y el espacio vivido serían los espacios de representación (el espacio de la imaginación, de lo simbólico, de la pasión y la acción; de lo sobreentendido) (Lefebvre, 2013).

\footnotetext{
8 Este recorrido se podría resumir en seis libros principales: El derecho a la ciudad (1969 [1968]), De lo rural a lo urbano (1971 [1970]), El pensamiento marxista y la ciudad (1973 [1972]), Espacio y política (1976b [1972]) y La producción del espacio (2013 [1974]). Pero también dos más: La proclamation de la comune (1965) y L'Irruption de Nanterre au sommet (1968).
}

En la tensión permanente (pugnas y resistencias) entre los distintos espacios, en el contexto de la sociedad capitalista domina el espacio concebido. Pero difícilmente el espacio vivido, donde se encuentran la pasión y la acción de la vida cotidiana, se somete a las reglas de la coherencia que las representaciones del espacio pretenden imponer. Lefebvre propone captar la experiencia cambiante de lo espacial a través de esta tensión trialéctica, reivindicando la potencialidad de los espacios de representación para actuar sobre las representaciones y las prácticas espaciales. En este sentido Lefebvre denuncia de forma muy clara la supuesta racionalidad (cientificidad en Blumenberg) del urbanismo como la expresión geográfica (instrumento) de las lógicas del mercado mundial y sus despiadadas consecuencias. «El espacio ordena, prescribe y proscribe» (Martínez Lorea, 2013: 17). Esto nos permite superar y evidenciar la heteronomización del espacio, es decir, la superación de un espacio que está fuera del alcance de los habitantes, de la abstracción fetichizada que lleva a los ciudadanos a abstraerse de sí mismos: reducido a quien asume los códigos, señales, prohibiciones e imposiciones del espacio percibido. Con esto aflora un aprendizaje impresionante, que el espacio no es un abstracto-instrumental, que nos aleja de las implicaciones que tienen las relaciones sociales en la producción (capital-trabajo-vida), ocultando las profundas desigualdades y contradicciones que genera. La realidad no se puede confundir con lo visible. El espacio, la ciudad, es una producción social, el escenario de nuestras vidas, el ambiente en el que se producen y reproducen nuestros sueños y la posibilidad de transformar la sociedad. 
Es soporte, pero también campo de acción. Esta aparente evidencia se camufla desde su propia concepción (desde el espacio concebido). La voluntad insaciable de control por parte de arquitectos, urbanistas y planificadores encaja muy bien con la pretensión expansiva y colonizadora de la circulación de capital a través del sector inmobiliario y el de la construcción, lo cual permite que de esta coincidencia de intereses se reduzca la ciudad a una cuestión técnica (no perteneciente al mundo de la vida) donde lo urbano no se piensa desde el conflicto de lo social, ni desde la potencia que las relaciones entre subjetividades que se pueden desplegar (replegar y plegar) mediante sus acuerdos, desacuerdos y formas espontáneas de vida, sino desde la utopía concebida de la ciudad moderna como mecanismo de homogeneización, como elusión de la ciudad. Sin espacio vivido no hay ciudad.

\section{BIBLIOGRAFÍA}

Blumenberg, H. (2013) Teoría del mundo de la vida. Buenos Aires: Fondo de Cultura Económica.

Cassirer, E. (1979) Filosofía de las formas simbólicas. México: Fondo de Cultura Económica.

Deleuze, G. (1989) El pliegue. Leibniz y el Barroco. Barcelona: Paidós.

Durán, L. (2014) ¿Es posible una fenomenología del mundo de la vida?, Investigaciones Fenomenológicas, 11: 309-323.

Follesdal, D. (1990) El concepto de Lebenswelt en Husserl (trad. Sergio Sánchez Benítez). En Hyder, D. y Rheinberger, H. (eds.) (2009) Science and the Life-World. Palo Alto: Standford University Press.

Husserl, E. (1985) Investigaciones lógicas. Madrid: Alianza [original: Husserliana XVIII]

Husserl, E. (1991) La crisis de las ciencias europeas y la fenomenología trascendental. Barcelona: Crítica. [original: Husserliana VI]
Lefebvre, H. (1965) La proclamation de la Comune. París: Gallimard.

Lefebvre, H. (1968) L'Irruption de Nanterre au sommet. París: Anthropos.

Lefebvre, H. (1969) El derecho a la ciudad. Barcelona: Península.

Lefebvre, H. (1971) Le Manifeste différentialiste. Paris: Gallimard.

Lefebvre, H. (1971) De lo rural a lo urbano. Barcelona: Península.

Lefebvre, H. (1972) La revolución urbana. Madrid: Alianza.

Lefebvre, H. (1973) El pensamiento marxista y la ciudad. México: Extemporáneos.

Lefebvre, H. (1976) Tiempos equívocos. Barcelona: Kairós.

Lefebvre, H. (1976b) Espacio y política. Barcelona: Península.

Lefebvre, H. (2013) La producción del espacio. Madrid: Capitán Swing. 
Lefebvre, H. (2014) Critique of everyday life. (Volume I, II, III). Londres: Verso

Martínez Lorea, I. (2013) Henri Lefebvre y los espacios de lo posible [prólogo]. En Lefebvre, H. (2013) La producción del espacio. Madrid: Capitán Swing.
Simmel, G. (1912) Die Religion. Frankfurt: Literarische Anstalt Rütten \& Loening [consultado en: http://www.digbib.org/ Georg_Simmel_1858/Die_Religion_.pdf]

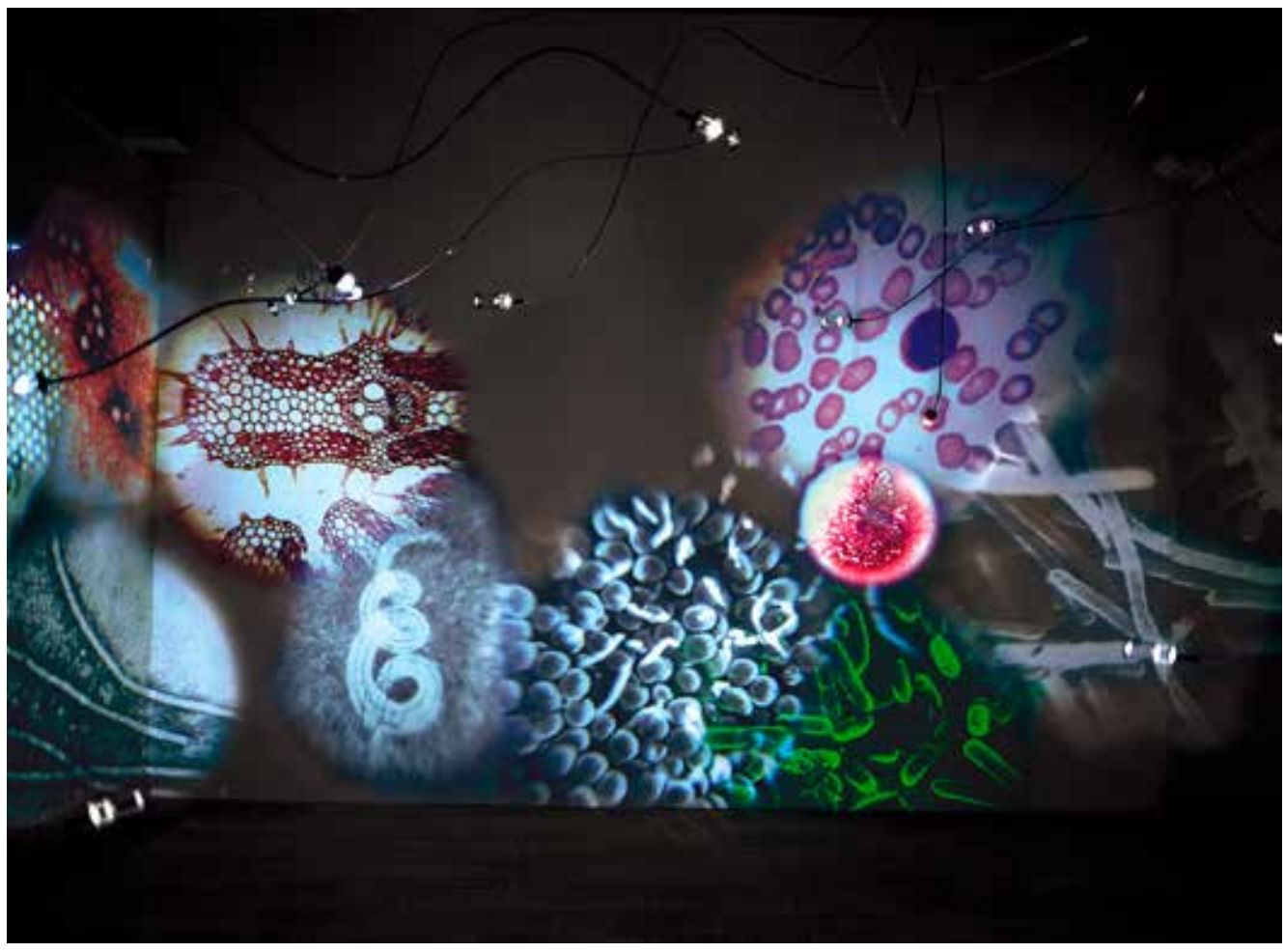

